\title{
Carpal tunnel syndrome related to antebrachial Cimino-Brescia fistula
}

\author{
A. E. HARDING AND J. LE FANU \\ From the Departments of Neurology and Nephrology, Royal Free Hospital, London
}

SUMMARY Two patients are described in whom symptoms of the carpal tunnel syndrome were provoked by haemodialysis for which an antebrachial arteriovenous fistula had been established. The symptoms in one case were partially relieved by ligation of the radial artery distal to the fistula, and in both they were abolished by decompression of the median nerve by section of the flexor retinaculum at the wrist. There is evidence that the median nerve is abnormally susceptible to ischaemia in the carpal tunnel syndrome, and it is suggested that in these patients the symptoms were provoked by a vascular steal mechanism related to the fistula.

The carpal tunnel syndrome arises from a wide variety of causes, and the most prominent symptom usually consists of painful nocturnal acroparaesthesiae in the affected limb. Warren and Otieno (1975) have recently reported the occurrence of symptoms considered to be due to the carpal tunnel syndrome during haemodialysis in patients with forearm arteriovenous fistulas. The present communication documents two such cases, in one of which the symptoms were partially relieved by ligation of the radial artery distal to the fistula. The symptoms were completely relieved by surgical decompression of the median nerve. The mechanism of origin of symptoms during dialysis is discussed.

\section{Case reports}

CASE 1

A 54 year old woman with the nephrotic syndrome was started on treatment by chronic haemodialysis in 1966 employing an arteriovenous shunt in the left arm. This was replaced in 1972 by a surgically produced arteriovenous fistula in the right forearm.

One year later she developed intermittent pain and paraesthesiae in the lateral three digits of the right hand, and noticed some difficulty with fine finger movements. Examination revealed wasting of the thenar eminence and cutaneous sensory im-

Address for correspondence and reprint requests: Dr A. E. Harding, Department of Neurology, Royal Free Hospital, Pond Street, London NW3 2QG, England.

Accepted 21 December 1976 pairment of median nerve distribution in the hand. Nerve conduction studies showed a borderline reduction in motor nerve conduction velocity in the forearm on recording from the abductor pollicis brevis $\left(45 \mathrm{~m} \mathrm{~s}^{-1}\right)$, with a slightly prolonged terminal latency $(5.5 \mathrm{~ms})$. No median nerve sensory action potential was detectable.

Temporary relief of symptoms was obtained by the local injection of prednisolone tertiary butyl acetate into the carpal tunnel. Surgical decompression was performed, but her symptoms recurred after six months, being particularly severe during dialysis. The pain decreased following ligation of the radial artery distal to the anastomosis, but weakness and sensory impairment persisted. Repeat nerve conduction studies at this stage showed that motor nerve conduction velocity over the forearm was $40 \mathrm{~m} \mathrm{~s}^{-1}$, with a now substantially prolonged terminal latency of $9 \mathrm{~ms}$.

Surgical exploration was again undertaken, and a thick fibrous band, producing narrowing of the nerve, was divided. This procedure led to relief of the patient's symptoms, which had not recurred at the time of her death one year later.

\section{CASE 2}

A 42 year old man presented with hypertension and chronic renal failure in 1972. Conservative management of his renal failure was unsuccessful and, therefore, haemodialysis with a CiminoBrescia fistula in the left arm was instituted. Eleven months later he complained of severe paraesthesiae, pain, and numbness within the distribution of the median nerve in the left hand. 
These symptoms occurred predominantly during dialysis. No abnormal neurological signs were detected.

The carpal tunnel was explored surgically, and the median nerve was noted to be hyperaemic. Decompression was performed by division of the flexor retinaculum with immediate relief of the patient's symptoms. These had not recurred by the time of his death nearly two years later.

\section{Discussion}

Warren and Otieno (1975) reported that 23 out of 36 patients using a forearm arteriovenous fistula for haemodialysis admitted to pain, numbness, and paraesthesiae in one or more of the lateral four digits of the hand on the side of the fistula during dialysis. No abnormal motor signs and no persisting sensory loss were detected, although in four patients, diminished tactile sensation of median nerve distribution was present immediately after dialysis, but disappeared within 24 hours. These symptoms were attributed to the carpal tunnel syndrome but this was not confirmed by nerve conduction studies. Lindstedt and Westling (1975) have also reported the occurrence of pain in the radial three fingers of the hand during haemodialysis employing an antebrachial CiminoBrescia arteriovenous fistula.

Warren and Otieno (1975) related the occurrence of symptoms attributed to median nerve dysfunction in their cases to oedema secondary to the increased venous pressure in the hand produced by the fistula. They found that predialysis venous pressure in the hand and hand volume were greater on the side of the fistula, and that hand volume increased during dialysis. This is an attractive suggestion which has been proposed as an important factor in the causation of nerve damage in the carpal tunnel syndrome by Sunderland (1976). Yet symptoms of the carpal tunnel syndrome are not a feature of other disorders that give rise to oedema of the arm and hand, such as axillary vein thrombosis or lymphoedema of the arm. Moreover, Fullerton (1963) found that in acute experiments in patients with the carpal tunnel syndrome, venous occlusion produced by a pressure cuff around the arm has no influence on nerve conduction, whereas inflation of the cuff above arterial pressure leads to a rapid failure of conduction.

An alternative suggestion is that the symptoms are provoked by ischaemia and related to a vascular 'steal' phenomenon caused by the fistula. Bussell et al. (1971) demonstrated by plethysmo- graphy that a radial-cephalic arteriovenous fistula constantly gives rise to increased forearm blood flow and reduced thumb blood flow. This was confirmed by Lindstedt and Westling (1975). They found that the disappearance of ${ }^{133}$ Xenon from the adductor pollicis was substantially reduced in patients with antebrachial arteriovenous fistulas. When flow through the fistula was prevented by digital compression, or when the radial artery distal to the fistula was occluded, the clearance of ${ }^{133}$ Xenon from the adductor pollicis showed an approximately fourfold increase.

Early discussions of the genesis of symptoms in the carpal tunnel syndrome considered the rossibilities of ischaemia (Simpson, 1956), and direct mechanical effects on nerve fibres (Thomas, 1960; Thomas and Fullerton, 1964). It seems likely that there is a dual mechanism for nerve damage (Fullerton, 1963). There is now good evidence for a direct mechanical effect on myelin (Neary et al., 1975), which presumably leads to a demyelinating conduction block and conduction delay. This process appears to be relatively independent of ischaemia, since fibres damaged in this way are not abnormally susceptible to ischaemia (Fullerton, 1963). They recover slowly when the nerve is decompressed (Goodman and Gilliatt, 1961). In $\stackrel{\circ}{\circ}$ contrast, it is known that ischaemia produced by the inflation of a pneumatic tourniquet around 8 the arm may reproduce the attacks of pain ando paraesthesiae (Gilliatt and Wilson, 1953), and give rise to a reversible conduction block. Gilliatt and Wilson (1954) found that median nerve sensory loss appeared with abnormal rapidity, and Fullerton (1963) demonstrated that conduction in motor fibres failed abnormally rapidly because of the development of a conduction block at the wrist. Pain and paraesthesiae are often relieved within 24 hours of carpal tunnel decompression, and this is paralleled by improvement in nerve conduction velocity (Hongell and Mattsson, 1971). In some instances improvement in sensory conduction is evident within 30 minutes. The rapidity of this recovery strongly suggests that relief of ischaemia is likely to be responsible.

These various observations, therefore, support the view that the attacks of pain and paraesthesiae in the carpal tunnel syndrome are the consequence of episodes of ischaemia. Thomas (1975) proposed that at times they may be due to partial obstruction of the brachial artery related to limb rosture during sleep. If the mechanism for the appearance of symptoms of median nerve dysfunction during haemodialysis in patients with antebrachial arteriovenous fistulas is a vascular 
steal, pre-existing median nerve compression in the carpal tunnel may render the patient more vulnerable to this complication. It is, therefore, of interest that both in the report by Lindstedt and Westling (1975) and in our first case, partial relief of symptoms was obtained by ligation of the radial artery distal to the arteriovenous fistula in the forearm, whereas complete relief of symptoms in both of our cases was obtained by surgical decompression of the median nerve at the wrist.

We wish to thank Professors R. W. Gilliatt and P. K. Thomas for helpful discussion, and Dr John Moorhead and Mr J. Chalmers for permission to publish details of cases 1 and 2 respectively.

\section{References}

Bussell, J. A.. Abbott, J. A., and Lim, R. C. (1971). A radial steal syndrome with arteriovenous fistula for haemodialysis. Annals of Internal Medicine, 75, 387-394.

Fullerton, P. M. (1963). The effect of ischaemia on nerve conduction in the carpal tunnel syndrome. Journal of Neurology, Neurosurgery, and Psychiatry, 26, 385-397.

Gilliatt, R. W.. and Wilson, T. G. (1953). A pneumatic tourniquet test in the carpal tunnel syndrome. Lancet, 2, 595-597.

Gilliatt, R. W., and Wilson, T. G. (1954). Ischaemic sensory loss in patients with peripheral nerve lesions. Journal of Neurology, Neurosurgery, and Psychiatry, 17, 104-114.

Goodman, H. V., and Gilliatt, R. W. (1961). The effect of treatment on median nerve conduction in patients with the carpal tunnel syndrome. Annals of Physical Medicine, 6, 137-155.

Hongell, A., and Mattsson, H. S. (1971). Neurographic studies before, after, and during operation for median nerve compression in the carpal tunnel syndrome. Scandinavian Journal of Plastic and Reconstructive Surgery, 5, 103-109.

Lindstedt, E., and Westling, H. (1975). Effects of an antebrachial Cimino-Brescia arteriovenous fistula on the local circulation in the hand. Scandinavian Journal of Urology and Nephrology, 9, 119-124.

Neary, D., Ochoa, J., and Gilliatt, R. W. (1975). Subclinical entrapment neuropathy in man. Journal of the Neurological Sciences, 24, 283-298.

Simpson, J. A. (1956). Electrical signs in the diagnosis of carpal tunnel and related syndromes. Journal of Neurology, Neurosurgery, and Psychiatry, 19, 275-280.

Sunderland, S. (1976). The nerve lesion in the carpal tunnel syndrome. Journal of Neurology, Neurosurgery, and Psychiatry, 39, 615-626.

Thomas, P. K. (1960). Motor nerve conduction in the carpal tunnel syndrome. Neurology (Minneapolis), 10, 1045-1050.

Thomas, P. K. (1975). In Peripheral Neuropathy, p. 506. Edited by P. J. Dyck, P. K. Thomas, and E. H. Lambert. W. B. Saunders: Philadelphia.

Thomas, P. K., and Fullerton, P. M. (1964). Nerve fibre size in the carpal tunnel syndrome. Journal of Neurology, Neurosurgery, and Psychiatry, 26, 520-527.

Warren, D. J., and Otieno, L. S. (1975). Carpal tunnel syndrome in patients on intermittent haemodialysis. Postgraduate Medical Journal, 51, 450-452. 